Case Report

Journal of Epilepsy Research pISSN 2233-6249 / eISSN 2233-6257

Received November 21, 2018

Revised December 11, 2018

Accepted December 20, 2018

Corresponding author:

Hea Ree Park, MD

Department of Neurology, Inje University

Ilsan Paik Hospital, Inje University College

of Medicine, 170 Juhwa-ro, Ilsanseo-gu,

Goyang 10380, Korea

Tel. +82-31-910-7276

Fax. $+82-31-910-7368$

E-mail; i0452@paik.ac.kr,

okokree@gmail.com

\title{
Endosulfan-Induced Prolonged Super-Refractory Status Epilepticus
}

\author{
Hea Ree Park, MD, Pamela Song, MD, Jae Jung Lee, MD, Joong-Yang Cho, MD, PhD \\ Department of Neurology, Inje University Ilsan Paik Hospital, Inje University College of Medicine, Goyang, Korea
}

Endosulfan is a highly toxic pesticide that causes hyperstimulation of the central nervous system by antagonizing gamma aminobutyric acid-mediated inhibition. Seizure is the most important manifestation of endosulfan poisoning, frequently progressing to status epilepticus and refractory status epilepticus. Here, we report a recent case of a 64-year-old man with endosulfan-induced super-refractory status epilepticus, which persisted for a remarkably longer period than has been described in previous reports. The patient arrived at the emergency room with continuous generalized tonic-clonic seizures. Electroencephalogram-recorded seizures that persisted even after intravenous administration of lorazepam and antiepileptic drugs. Intravenous anesthetic agents were administered for 9 days to confront the persistently recurring seizures. Immediately after this treatment period, the seizures subsided, and the patient showed marked neurological improvement. After 2 months however, he died of multiple systemic complications. This case report elucidates the importance of aggressive evaluation and management including continuous EEG monitoring in cases of endosulfan-related status epilepticus. (2018;8:93-96)

Key words: Endosulfan poisoning, Endosulfan-induced super-refractory status, Status epilepticus, Seizure, Intoxication

\section{Introduction}

Endosulfan (hexachloro-hexahydro-methano-benzodioxathiepinoxide) is an organochlorine insecticide that belongs to the class of cyclodienes. ${ }^{1}$ Endosulfan exerts its toxicity in humans by causing central nervous system (CNS) hyperstimulation through the antagonism of gamma aminobutyric acid-mediated inhibition. ${ }^{2}$ Seizure is the most common neurological complication of endosulfan poisoning, with an incidence rate $70-92 \% .^{3-6}$ In contrast to most drug-induced seizures that are usually self-limited, endosulfan-related seizures are highly persistent. ${ }^{7}$ Previous studies revealed that $46-78 \%$ of acute endosulfan poisoning cases presented status epilepticus (SE). ${ }^{4,8}$ Seizure duration of endosulfan-related SE varies among studies. ${ }^{3-5,8} \mathrm{~A}$ recent study reported that median duration of antiepileptic drug (AED) use in endosulfan poisoning was 48.0 hours (range, 29.3-103.5 hours) in non-refractory SE and 48.5 hours (range, 23.8-63.5 hours) in refractory SE (RSE), and another study reported seizure durations of up to 23.2 hours. ${ }^{4,8}$ Here, we report a case of endosulfan-induced super RSE (SRSE), which persisted for a remarkably longer period than what was reported in other cases.

\section{Case}

A 64-year-old man without any underlying disease arrived at the emergency room (ER) with continuous generalized tonic-clonic seizures after ingesting a pesticide labeled as endosulfan. The exact amount of endosulfan intake could not be verified. Soon after admission, the patient had a cardiac arrest, from which he recovered after 10 minutes of resuscitations. Initial blood tests demonstrated severe acidosis (pH 6.80) and elevated lactate concentrations ( $242.5 \mathrm{mg} / \mathrm{dL}$ ) with normal liver and kidney function. Serum concentration of neuron-specific enolase (NSE) was not measured after resuscitation. Brain computed tomography revealed no abnormalities, whereas brain magnetic resonance imaging (MRI) was not performed due to instability of the patient's vital signs. The patient was treated promptly with the established SE protocol; however, the generalized tonic-clonic seizures did not subside. Subsequently, the patient received intravenous (IV) administrations of vigorous anticonvulsants including $14 \mathrm{mg}$ of lorazepam, 1,400 mg of valproic acid, and 1,500 $\mathrm{mg}$ of levetiracetam. Continuous electroencephalography (EEG) data revealed continuous generalized EEG seizures.

He was then administered status epilepticus IV anesthetic agent

This is an Open Access article distributed under the terms of the Creative Commons Attribution Non-Commercial License (http://creativecommons.org/licenses/by-nc/3.0/) which permits unrestricted non-commercial use, distribution, and reproduction in any medium, provided the original work is properly cited. 
therapy starting with midazolam. Clinical seizures subsided when midazolam dose was increased to $2 \mathrm{mg} / \mathrm{kg}$ hours, 4 hours after admission to the ER. However, EEG seizures persisted even after clinical seizures had subsided; therefore, IV propofol was administered. After 24 hours, the propofol dose was increased to $4 \mathrm{mg} / \mathrm{kg}$ hours and EEG data revealed burst suppression with a few breach-through seizures, each lasting less than 4 seconds. After propofol administration at a maximum dose for 2 days, propofol infusion syndrome occurred causing rhabdomyolysis, renal failure, abnormal cardiac function, and fluctuations in blood pressure and heart rate. Propofol was then switched to thiopental continuous infusion. During the following seven days of coma therapy, his EEG data presented seizures arising from the left or right fronto-temporal area when IV anesthetic agents were reduced, but stabilized to burst suppression or continuous slow when IV anesthetic agents were increased (Fig. 1).

IV anesthesia was administered for a total of 9 days, after which

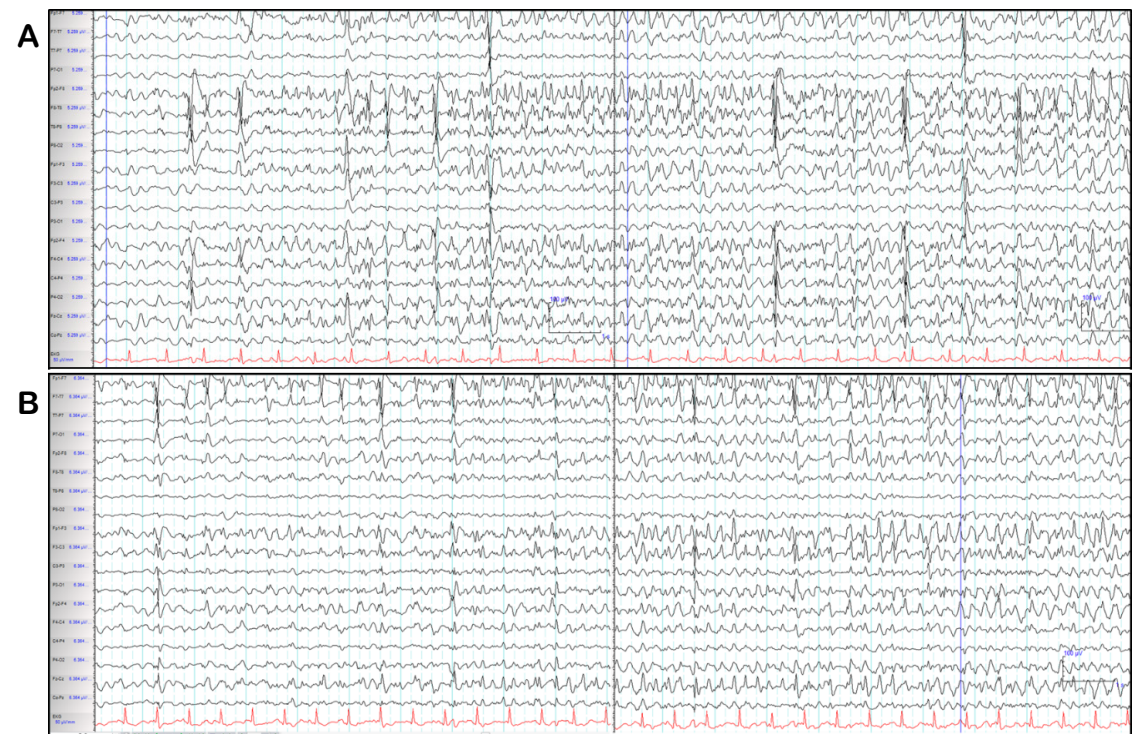

Figure 1. Electroencephalogram (EEG) showing seizures arising from the right or left fronto-temporal area. (A) The EEG seizure starts as repetitive sharp waves at the right anterior temporal area evolving to irregular theta rhythm and then to spike-wave mixture at right fronto-temporal areas where they were recorded. (B) Periodic sharp waves at the left anterior temporal areas were observed which progressed to a spike-wave mixture at the left frontal area and irregular theta rhythm in the left hemisphere.

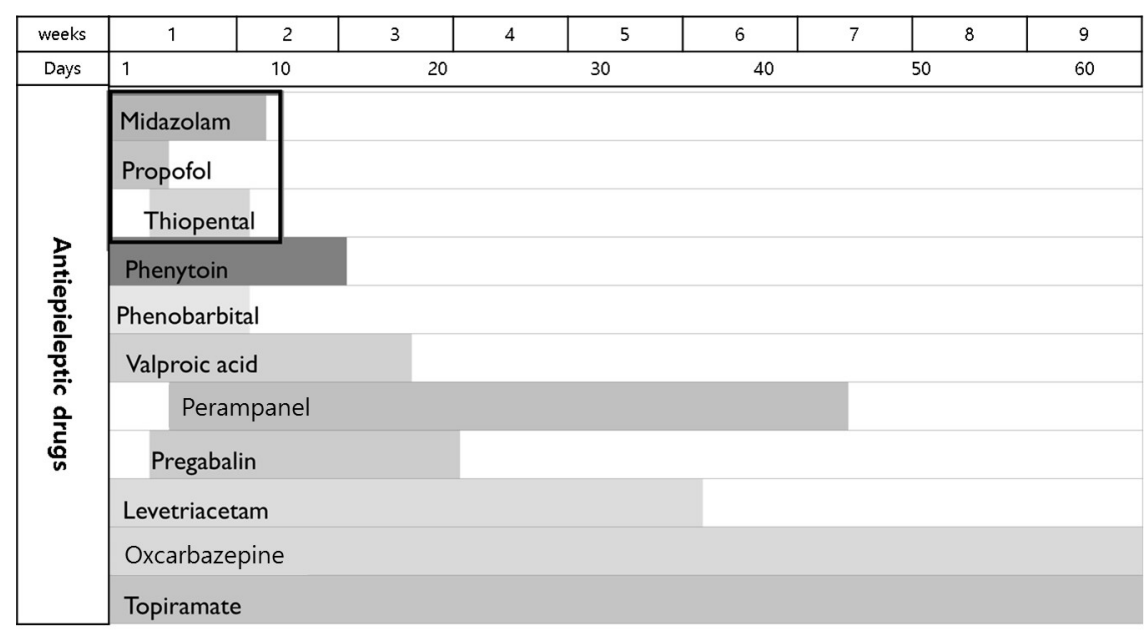

Figure 2. Scheme of the multiple antiepileptic drugs, including intravenous anesthetic agents (box), administered to the patient during hospitalization. The numbers in the top panel represent hospital weeks and days, respectively. 
the patient's EEG seizures subsided. During coma therapy, the patient presented with aspiration pneumonia, ileus, ischemic colitis, renal failure, and pulmonary edema. After cessation of IV anesthetic agent administration, multiple AED were applied to control the patient's seizures and were adjusted to his medical condition which included liver dysfunction and renal failure. Fig. 2 summarizes the record of the multiple AED used in this case during hospitalization. For 7 weeks after admission, the patient was in the intensive care unit with tracheostomy and mechanical ventilation. Following tapering of IV anesthetic agents, he showed remarkable neurological improvement, exhibiting eye contact, and responding optimally to simple obey commands. Despite this neurological improvement, the patient died of ischemic colitis on hospital day 62.

\section{Discussion}

In the present case, endosulfan-induced seizures progressed to SRSE and persisted for a markedly long period. The patient was subjected to 9-day coma therapy and 2-month AED treatment. A recent study reported that the progression rates of endosulfan poisoning-related seizures to SE and RSE were $78.1 \%$ and $54.4 \%$, respectively, ${ }^{4}$ considerably higher than that of other drug-induced seizures, reported to reach $10 \% .{ }^{9}$ This could be attributed to the high lipid solubility of endosulfan; despite its short half-life in the plasma, endosulfan is rapidly absorbed, quickly reaches tissues such as the CNS, and then is slowly released from its lipophilic depots. ${ }^{10}$ The half-life of endosulfan and its metabolites is estimated to be 1-7 days. ${ }^{11}$

In previous reports, the maximum seizure duration was 23.2 hours. ${ }^{8}$ Recently, one study reported a median seizure duration of AED use without seizure recurrence of 48.5 hours (range, 23.8-63.5) in RSE, but the duration of the seizures was not evaluated. Seizure duration is reported as an important prognostic factor in acute endosulfan poisoning. ${ }^{3-5,8,10}$ However, previous data on seizure duration are limited, in that they only included the duration of convulsive seizures (since continuous EEG monitoring was not applied in most of the cases). This is related to the fact that pesticide poisoning most commonly occurs in rural communities without adequate EEG resources. Therefore, previous research may have underestimated seizure duration because non-convulsive SE, following convulsive seizures was not assessed. In the present case, seizure duration was strikingly longer than that observed in previous reports and this could be associated with the fact that we still recorded EEG seizures through continuous EEG monitoring even after clinical seizures had subsided. Therefore, non-convulsive seizures induced by endosulfan poisoning could persist for crucially longer periods than what has been reported in previous studies, and thus, EEG monitoring would be essential in endosulfan-related SE even after cessation of convulsive seizures.

It has been reported that patients with endosulfan poisoning also manifested hepatic, renal and cardiac toxicities along with common complications such as rhabdomyolysis, renal failure, hypotension, nausea, vomiting, and metabolic derangement. ${ }^{6,8,12}$ These systemic complications occurred more frequently in cases of RSE, and were associated with high mortality of endosulfan-related RSE. In the present case, various medical complications occurred including rhabdomyolysis, renal failure, abnormal cardiac function, aspiration pneumonia, ischemic colitis, and pulmonary edema. These complications were presumably induced by the prolonged seizures, hemodynamic instability and administration of various AED, including IV anesthetic agents as well as directly by the toxicity of endosulfan. This strengthens the importance of general medical management in endosulfan poisoning. Although the patient of the present case showed marked neurological recovery signs such as displaying eye contact and simple obey commands, he did not survive because of other health issues including hepatotoxicity, nephrotoxicity, and ischemic colitis.

Endosulfan was globally banned under the Stockholm Convention in May 2011 because of its threat to human health and the environment. Since 2011, agricultural chemical registration of endosulfan has been cancelled in South Korea. However, endosulfan poisoning still occurs due to stocks of endosulfan that existed prior to the implementation of the prohibition. One study reported admitting six cases of acute endosulfan poisoning in a single hospital between 2014 and 2016 . $^{4}$ In the present case, acute poisoning by endosulfan occurred in May 2017, implicating that clinicians should focus on the management of endosulfan intoxication even if it was phased out in the market.

This case had several limitations. First, the amount of endosulfan ingested was not assessed. Although one study reported the ingestion of $>100 \mathrm{~mL}$ of endosulfan as risk factor for death, most previous studies have not assessed the amount of endosulfan ingestion because family or colleagues might not have been aware, and also the ingested quantity could not be estimated by using the actual absorbed amount due to loss from vomiting. Second, the possibility of combined hypoxic brain damage-related SE could not be excluded. Cardiac arrest occurred after seizure onset, recovery after 10 minutes of resuscitation, then persistent seizure returned for 9 days. Prognostic markers after 
cardiac arrest such as serum NSE or brain MRI were not assessed, therefore, the evaluation of the severity of hypoxic brain damage was limited. However, if the hypoxic brain damage was the main cause of SE, the patient's neurological reversibility after controlling of SE would not be explained.

In conclusion, endosulfan-related SRSE may persist for remarkably longer periods than what has been stated in previous reports, requiring IV administration of anesthetic agents for more than a week. Aggressive evaluation and management including continuous EEG monitoring, IV administration of AEDs and anesthetic drugs should be considered together with general medical care in cases of endosulfan-related SE.

\section{References}

1. Menezes RG, Qadir TF, Moin A, et al. Endosulfan poisoning: an overview. J Forensic Leg Med 2017;51:27-33.

2. Silva MH, Beauvais SL. Human health risk assessment of endosulfan. I: toxicology and hazard identification. Regul Toxicol Pharmacol 2010;56: 4-17.
3. Han $B G$, Lee JH, Lee KW. Effect of seizure on prognosis in acute endosulfan intoxication. J Korean Soc Clin Toxicol 2009;7:77-82.

4. Moon JM, Chun BJ, Lee SD. In-hospital outcomes and delayed neurologic sequelae of seizure-related endosulfan poisoning. Seizure 2017;51:43-9.

5. Moon JW, Moon JM, Lee BK, Roo HH. Prognostic predictors of endosulfan intoxication. J Korean Soc Emerg Med 2009;20:185-91.

6. Karatas A, Aygun D, Baydin A. Characteristics of endosulfan poisoning: a study of 23 cases. Singapore Med J 2006;47:1030-2.

7. Chen HY, Albertson TE, Olson KR. Treatment of drug-induced seizures. Br J Clin Pharmacol 2016;81:412-9.

8. Kim SE, Kim Sl, Lee JB, et al. Clinical analysis of acute endosulfan poisoning: single center experience. J Korean Soc Clin Toxicol 2015;13:71-7.

9. Thundiyil JG, Rowley F, Papa L, Olson KR, Kearney TE. Risk factors for complications of drug-induced seizures. J Med Toxicol 2011;7:16-23.

10. Yavuz $Y$, Yurumez $Y$, Kücüker $H$, Ela $Y$, Yüksel $S$. Two cases of acute endosulfan toxicity. Clin Toxicol (Phila) 2007;45:530-2.

11. Lee I, Eriksson P, Fredriksson A, Buratovic S, Viberg H. Developmental neurotoxic effects of two pesticides: behavior and neuroprotein studies on endosulfan and cypermethrin. Toxicology 2015;335:1-10.

12. Yadla M, Yanala SR, Parvithina S, Chennu KK, Annapindi N, Vishnubhotla S. Acute kidney injury in endosulfan poisoning. Saudi J Kidney Dis Transp/ 2013;24:592-3. 